M. Shimura

Nagoya Math. J.

Vol. 95 (1984), 105-116

\title{
A LIMIT THEOREM FOR TWO-DIMENSIONAL CONDITIONED RANDOM WALK
}

\author{
MICHIO SHIMURA
}

\section{§1. Introduction}

Let $\left\{\left(S_{n}, T_{n}\right), n=0,1,2, \cdots\right\}$ be a two-dimensional random walk with stationary independent increments starting at the origin $\mathbf{0}$. Throughout the paper we always assume the following condition:

$$
E S_{1}=E T=0 \text { and the covariance matrix of }\left(S_{1}, T_{1}\right) \text { is }\left(\begin{array}{ll}
1 & 0 \\
0 & 1
\end{array}\right) .
$$

We set a probability space $(\Omega, \mathscr{A}, P)$ on which all our random quantities are defined. Let $D$ stand for the family of right continuous functions $z$ on $[0, \infty)$ with left-hand limits, and with $z(0)=\mathbf{0}$. Let $\mathscr{D}$ be the usual $\sigma$-field of $D$. We define a sequence of random elements $Z_{n}(t)=\left(X_{n}(t)\right.$, $\left.Y_{n}(t)\right), n=1,2, \cdots$, in $D$ by

$$
Z_{n}(t)=n^{-1 / 2}\left(S_{k}, T_{k}\right) \text { for } k / n \leqq t<(k+1) / n \text { and } k=0,1,2, \cdots .
$$

Let $\{Z(t)=(X(t), Y(t)), t \geqq 0\}$ be the two-dimensional standard Brownian motion with continuous paths starting at the origin. Let $\mathscr{E}(Z)$ donote the random set of all finite open intervals $(\tau, v)$ in $[0, \infty)$ such that

$$
Y(t) \geqq Y(\tau) \quad \text { for } 0 \leqq t \leqq \tau, \quad Y(t)>Y(\tau) \quad \text { for } \tau<t<v \quad \text { and } \quad Y(\tau)=Y(\nu) .
$$

Here we note the following fact. We set

$$
\bar{Y}(t)=Y(t)-\min _{0 \leqq s \leqq t} Y(s) \quad \text { and } \quad \bar{Z}(t)=(X(t), \bar{Y}(t)) \text { for } t \geqq 0 .
$$

Then the process $\bar{Z}(\cdot)$ is the reflecting Brownian motion on the upper halfplane with $\bar{Z}(0)=\mathbf{0}$, and $\mathscr{E}(Z)$ coincides with the set of excursion intervals of the $\bar{Z}(\cdot)$.

Let $\sigma_{F}$ be the exit time from a closed domain $F$ in $R^{2}$ defined by

$$
\sigma_{F}(z)=\inf \{t ; z(t) \notin F\} \quad \text { for } z \in D
$$

Received July 12, 1983. 
(inf $\phi=\infty$ ). We are interested in the weak convergence of conditional probabilities $P\left(Z_{n} \in{ }^{*} \mid \sigma_{F}\left(Z_{n}\right)>1\right), n=1,2, \cdots$, on $(D, \mathscr{D})$ under the assumption:

$$
\text { the boundary } \partial F \text { of } F \text { contains the origin. }
$$

Let us state our main result. Let $h \in \boldsymbol{C}(\boldsymbol{R} \rightarrow[0, \infty))$ satisfy the following assumption:

$$
h(0)=0 \text { and } h(x)>0 \text { on }[-b, b]-\{0\} \text { for some } b>0 .
$$

For such $h$ we define $F_{h}=\{(x, y) ; y \geqq h(x)\}$, which clearly satisfies (1.2). Let $d$ be the metric on $\boldsymbol{D}$ which induces the extended $J_{1}$ topology (Lindvall [7], p. 115). We set $F_{0}=\boldsymbol{R} \times[0, \infty)$, the upper half-plane, and introduce another metric $\stackrel{d}{d}$ on $D$ by

$$
\stackrel{\circ}{d}\left(z_{1}, z_{2}\right)=d\left(z_{1}, z_{2}\right)+\left|\sigma_{F_{0}}\left(z_{1}\right)-\sigma_{F_{0}}\left(z_{2}\right)\right|,
$$

where we understand $\infty-\infty=0$. Let $(\hat{\tau}, v)$ be the first element of $\mathscr{E}(Z)$ satisfying $v-\tau>1$ (note that $(\hat{\tau}, \hat{v})$ exists almost surely), and define the process $\hat{Z}$ by

$$
\hat{Z}(t)=Z(\hat{\tau}+t)-Z(\hat{\tau}) \quad \text { for } t \geqq 0 .
$$

We will prove the following theorem.

TheOREm. Assume that $h \in \boldsymbol{C}(\boldsymbol{R} \rightarrow[0, \infty))$ satisfies (1.3) and the following assumption:

$$
\begin{aligned}
& |x|^{-1} h(x) \text { is nondecreasing on }(0, b] \text {, nonincreasing on }[-b, 0) \text { and } \\
& |x|^{-1} h(x) \rightarrow 0 \text { as } x \rightarrow 0 \text {, }
\end{aligned}
$$

and the integral

$$
\int_{0<|x|<1}|x|^{-2} h(x) d x
$$

converges. Then the sequence of conditional probabilities $P\left(Z_{n} \in{ }^{*} \mid \sigma_{F_{n}}\left(Z_{n}\right)\right.$ $>1), n=1,2, \cdots$, converges weakly to $P\left(\hat{Z} \in * \mid \sigma_{F_{n}}(\hat{Z})>1\right)$ in $(D, \stackrel{d}{d})$.

Theorem is a two-dimensional analogue (viz conditioned to stay on $F_{h}$ ) of the limit theorem of one-dimensional random walk conditioned to stay positive which has been considered by Iglehart [4], Bolthausen [2], Shimura [11] and others. First we observe the following two-dimensional version (viz conditioned to stay on the half-plane) of the above onedimensional conditioned limit theorem: 


$$
P\left(Z_{n} \in * \mid \sigma_{F_{0}}\left(Z_{n}\right)>1\right) \stackrel{w}{\longrightarrow} P(\hat{Z} \in *) \quad \text { in }(D, \stackrel{\circ}{d}),
$$

where $\stackrel{w}{\longrightarrow}$ denotes the weak convergence. In Section 2 we give a proof of (1.6), then, in Section 3, we consider a necessary and sufficient condition for $h$ under which $P\left(\sigma_{F_{h}}(\hat{Z})>1\right)>0$, that is, the origin is an irregular point to the set $\left(F_{h}\right)^{c}$ for the limit process $\hat{Z}$. Difficulty in proving Theorem consists in the fact that $\sigma_{F_{h}}(\cdot)$ is $P(\hat{Z} \in *)$-almost surely discontinuous and that, for this reason, (1.6) does not imply Theorem. The most essential point in our proof is to overcome the difficulty. It is done by obtaining the following estimate:

$$
\lim _{\dot{\delta} \rightarrow 0+} \limsup _{n \rightarrow \infty} P\left(\sigma_{F_{h}}\left(Z_{n}\right) \leqq \delta \mid \sigma_{F^{\prime}}\left(Z_{n}\right)>1\right)=0,
$$

see Section 4, most of which is devoted to this estimate. In Section 5 we consider an asymptotic behavior of moments for the conditioned random walk in (1.6) and get an estimate (4.14), which plays a key role in showing (1.7).

Acknowledgement. The author is grateful to the referee who helped him to improve the original manuscript.

\section{§2. Proof of (1.6)}

The idea of the proof given here is due to Bolthausen [2] (refer also to [11] for the detail). For any fixed $n$, let $\lambda_{m}, m=0,1,2, \cdots$, be a sequence of "decreasing ladder epochs" of the process $Z_{n}(\cdot)$ defined by

$$
\lambda_{0}=0 \quad \text { and } \quad \lambda_{m}=\inf \left\{t>\lambda_{m-1} ; Y_{n}(t)<Y_{n}\left(\lambda_{m-1}\right)\right\}, \quad m=1,2, \cdots .
$$

Let $\hat{m}=\min \left\{m ; \lambda_{m+1}-\lambda_{m}>1\right\}$, which is finite almost surely, and set

$$
\hat{\tau}_{n}=\lambda_{\hat{m}}, \hat{v}_{n}=\lambda_{\hat{m}+1} \quad \text { and } \quad \hat{Z}_{n}(t)=Z_{n}\left(\hat{\tau}_{n}+t\right)-Z_{n}\left(\hat{\tau}_{n}\right) \text { for } t \geqq 0 .
$$

Then we easily get the following formula:

$$
P\left(\left(Z_{n}, \sigma_{F_{0}}\left(Z_{n}\right)\right) \in L \mid \sigma_{F_{0}}\left(Z_{n}\right)>1\right)=P\left(\left(\hat{Z}_{n}, \hat{v}_{n}-\hat{\tau}_{n}\right) \in L\right)
$$

for every measurable set $L$ in $D \times R$. Noting $Z_{n} \stackrel{w}{\longrightarrow} Z$ in $(D, d)$ by the central limit theorem, we apply the continuous mapping theorem (Billingsley [1], Theorem 5.5) on the right-hand side of (2.1) in the same way as [2] to get

$$
P\left(\left(\hat{Z}_{n}, \hat{v}_{n}-\hat{\tau}_{n}\right) \in *\right) \stackrel{w}{\longrightarrow} P((\hat{Z}, \hat{v}-\hat{\tau}) \in *) \text { as } n \longrightarrow \infty
$$


in the product space $(\boldsymbol{D}, d) \times \boldsymbol{R}$. Hence we have (1.6).

\section{§3. Kolmogorov type test for the Brownian excursions}

We first show the following lemma.

Lemma 3.1. Suppose that $h \in \boldsymbol{C}(\boldsymbol{R} \rightarrow[0, \infty))$ satisfies (1.4) in Section 1. Then we have

$$
P\left(\sigma_{F_{h}}(Z(\tau+\cdot)-Z(\tau))>0 \text { for all }(\tau, \nu) \in \mathscr{E}(Z)\right)=1
$$

or

$$
P\left(\sigma_{F_{h}}(Z(\tau+\cdot)-Z(\tau))=0 \text { for all }(\tau, \nu) \in \mathscr{\varrho}(Z)\right)=1
$$

according as the integral (1.5) converges or diverges.

Proof. Let $\left(\tau^{(n, k)}, v^{(n, k)}\right)$ be the $k$-th element of

$$
\mathscr{E}_{1 / n}(Z)=\left\{(\tau, v) \in \mathscr{E}(Z) ; \max _{\tau \leqq t \leqq \nu} \bar{Y}(t) \geqq 1 / n\right\},
$$

and put $Z^{(n, k)}(t)=Z\left(\tau^{(n, k)}+t\right)-Z\left(\tau^{(n, k)}\right)$ for $t \geqq 0$. Clearly $\left\{Z^{(n, k)}, k=\right.$ $1,2, \cdots\}$ is a sequence of identically distributed processes. Hence, noting $\mathscr{E}_{1 / n}(Z) \uparrow \mathscr{E}(Z)$ as $n \rightarrow \infty$, we have that (3.1) or (3.2) is equivalent to

$$
P\left(\sigma_{F_{h}}\left(Z^{(n, 1)}\right)>0\right) \equiv 1 \quad \text { or } \quad 0 \quad \text { for every } n=1,2, \cdots,
$$

respectively.

Let $W(\cdot)$ be a three-dimensional standard Brownian motion independent of $X(\cdot)$. Note that the radial process $\{\|W(t)\|, t \geqq 0\}$ is the threedimensional Bessel process. We set $\rho_{a}(z)=\inf \{t ; y(t) \geqq a\}$ for $z=(x, y)$ $\in C\left([0, \infty) \rightarrow R^{2}\right)$, and consider two killed processes

$$
\stackrel{\circ}{Z}^{(n, 1)}=\left\{\boldsymbol{Z}^{(n, 1)}(t), 0 \leqq t<\rho_{1 / n}\left(\boldsymbol{Z}^{(n, 1)}\right)\right\}
$$

and

$$
\stackrel{i}{U}=\left\{U(t)=(X(t),\|W(t)\|), 0 \leqq t<\rho_{1 / n}(U)\right\} .
$$

Then it follows from Williams [13] that the processes $\dot{Z}^{(n, 1)}$ and $\dot{U}^{\circ}$ are identical in law. Combining this fact with (3.3), we reduce the lemma to Ito and McKean [5], 7.11, 9).

We note that Brownian motion has absolutely continuous transition probability. Then, using the above identification of $\dot{Z}^{(n, 1)}$ with $\dot{U}^{\circ}$, we obtain $P\left(Z^{(n, 1)}(s) \in \partial F_{h}\right)=0$ for every $n=1,2, \cdots$ and $s>0$. Hence we have the following lemma. 
Lemma 3.2. Let $h \in C(R \rightarrow[0, \infty))$. Then we have $P\left(Z(\tau+s)-Z(\tau) \in \partial F_{h} \quad\right.$ for some $\left.(\tau, \nu) \in \mathscr{E}(Z)\right)=0$ for every $s>0$.

\section{§. Proof of Theorem}

We note the following identity

$$
P\left(Z_{n} \in L \mid \sigma_{F_{h}}\left(Z_{n}\right)>1\right)=\frac{P\left(Z_{n} \in L ; \sigma_{F_{h}}\left(Z_{n}\right)>1 \mid \sigma_{F_{0}}\left(Z_{n}\right)>1\right)}{P\left(\sigma_{F_{h}}\left(Z_{n}\right)>1 \mid \sigma_{F_{0}}\left(Z_{n}\right)>1\right)}
$$

for $L \in \mathscr{D}$. Since $P\left(\sigma_{F_{h}}(\hat{Z})>1\right)>0$ by Lemma 3.1, and since

$$
P\left(\sigma_{F_{h}}(\hat{Z})=1\right) \leqq P\left(\hat{Z}(1) \in \partial F_{h}\right)=0
$$

by Lemma 3.2, we can conclude

$$
P\left(Z_{n} \in L \mid \sigma_{F_{h}}\left(Z_{n}\right)>1\right) \longrightarrow P\left(\hat{Z} \in L \mid \sigma_{F_{h}}(\hat{Z})>1\right)
$$

for every $P(\hat{Z} \in *)$-continuity set $L$, if we have the following refinement of (1.6).

Lemma 4.1. If $h \in \boldsymbol{C}(\boldsymbol{R} \rightarrow[0, \infty))$ satisfies the condition in Theorem, we have

$$
P\left(\left(Z_{n}, \sigma_{F_{h}}\left(Z_{n}\right)\right) \in * \mid \sigma_{F_{0}}\left(Z_{n}\right)>1\right) \stackrel{w}{\longrightarrow} P\left(\left(\hat{Z}, \sigma_{F_{h}}(\hat{Z})\right) \in *\right)
$$

in $(D, \stackrel{\circ}{d}) \times R$.

Proof. (First Step) Let $Q_{n}(*)=P\left(Z_{n} \in *\right)$ denote the induced measure on $(D, \mathscr{D})$. We show that (4.1) is proved if we have (1.7), that is,

$$
\lim _{\delta \rightarrow 0+} \limsup _{n \rightarrow \infty} Q_{n}\left(\sigma_{F^{\prime}} \leqq \delta \mid \sigma_{F_{0}}>1\right)=0,
$$

in addition to (1.6). To do this we introduce an auxiliary time $\sigma_{\delta}(z)=$ $\inf \left\{t \geqq \delta ; z(t) \notin F_{h}\right\}$ for $\delta>0$. We note that $\sigma_{\delta}(\cdot)$ is a continuous mapping. For $0<\delta<u$,

$$
\begin{aligned}
& Q_{n}\left(L ; \sigma_{\delta} \leqq u \mid \sigma_{F_{0}}>1\right) \leqq Q_{n}\left(L ; \sigma_{F_{h}} \leqq u \mid \sigma_{F_{0}}>1\right) \\
& \quad \leqq Q_{n}\left(L ; \sigma_{\delta} \leqq u \mid \sigma_{F_{0}}>1\right)+Q_{n}\left(\sigma_{F_{h}} \leqq \delta \mid \sigma_{F_{0}}>1\right)
\end{aligned}
$$

We first let $n \rightarrow \infty$, and then $\delta \rightarrow 0+$ for $P(\hat{Z} \in *)$-continuity set $L$. Since $\tau_{\delta} \rightarrow \sigma_{F_{h}}$ as $\delta \rightarrow 0+$, we have from (1.6) and (4.2) that both the extreme terms in the above inequality tend to $P\left(\hat{Z} \in L ; \sigma_{F_{h}}(\hat{Z}) \leqq u\right)$. This proves (4.1).

Furthermore, in proving (4.2), we may assume without loss of generality the following: 


$$
\begin{aligned}
& \psi(x)=|x|^{-1} h(x) \text { is bounded on } R, \text { nondecreasing on }(0, \infty) \text { and } \\
& \text { nonincreasing on }(-\infty, 0),
\end{aligned}
$$

where we define $\psi(0)=0$. Indeed we have the following fact:

Suppose that $h \in C(R \rightarrow[0, \infty))$ satisfies (1.3) and (1.4). Put $h_{1} \in C(R \rightarrow$ $[0, \infty))$ to satisfy $h_{1}(x)=h(x)$ on $[-b, b]$ and (4.3). If $h_{1}$ satisfies (4.2), then so does $h$.

Let $B$ be the closed disc of radius $b$ with the centre $\mathbf{0}$. Then we have

$$
Q_{n}\left(\sigma_{F_{n}} \leqq \delta \mid \sigma_{F_{0}}>1\right) \leqq Q_{n}\left(\sigma_{F_{1}} \leqq \delta \mid \sigma_{F_{0}}>1\right)+Q_{n}\left(\sigma_{B} \leqq \delta \mid \sigma_{F_{0}}>1\right)
$$

for $\delta>0$. Since $\sigma_{B}(\cdot)$ is a continuous mapping, we easily conclude the above fact from (1.6).

(Second Step) We prove (4.2) under the assumption (4.3). Set $\mathscr{S}_{k}=$ $\left|S_{k}\right| \psi\left(n^{-1 / 2} S_{k}\right)$ (the suffix $n$ in $\mathscr{S}_{k}$ is suppressed). Let $N(T)=\min \{k$; $\left.T_{k}<0\right\}$. For any fixed positive integer $k_{0}$, we have

$$
Q_{n}\left(\sigma_{F_{h}} \leqq \delta \mid \sigma_{F_{0}}>1\right) \leqq\left\{I_{1}\left(k_{0}\right)+I_{2}\left(k_{0}, \delta n\right)\right\} / P(N(T)>n),
$$

where

$$
I_{1}\left(k_{0}\right)=P\left(T_{k}<\mathscr{S}_{k}, 0<{ }^{\exists} \leqq k_{0} ; N(T)>n\right),
$$

and

$$
I_{2}\left(k_{0}, \delta n\right)=P\left(T_{k}<\mathscr{S}_{k}, k_{0}<{ }^{\sharp} \leqq \delta n ; N(T)>n\right) .
$$

Hence (4.2) is proved if we have

$$
\lim _{n \rightarrow \infty}\left\{I_{1}\left(k_{0}\right) / P(N(T)>n)\right\}=0 \quad \text { for every } k_{0}
$$

and

$$
I_{2}\left(k_{0}, \delta n\right) / P(N(T)>n) \leqq f(\delta)+K k_{0}^{-1 / 2},
$$

where $f(\delta)$ is independent of $k_{0}$ and $n$, and satisfies $f(\delta) \rightarrow 0$ as $\delta \rightarrow 0+$, and $K$ is a positive constant not depending on $k_{0}, n$ and $\delta$.

Before proving (4.4) and (4.5), we introduce useful tail estimate of the absorption time $N\left(T^{y}\right)=\min \left\{k ; T_{k}+y<0\right\}$ :

For each $y \geqq 0$ and $n=1,2, \cdots$, we have

$$
\kappa_{1} n^{-1 / 2} \leqq P\left(N\left(T^{y}\right)>n\right) \leqq \kappa_{2}(y+1) n^{-1 / 2},
$$

where $\kappa_{1}$ and $\kappa_{2}$ are positive constants. 
The first estimate in (4.6) is an easy consequence of the formula

$$
P(N(T)>n) \sim e^{\mu}(\pi n)^{-1 / 2} \quad(n \longrightarrow \infty),
$$

where $\mu$ is given by the convergent series

$$
\mu=\sum_{n=1}^{\infty} n^{-1}\left\{P\left(T_{n} \geqq 0\right)-1 / 2\right\}
$$

'Spitzer [12]). The second one is given by Kozlov [6].

For $A_{1}, \cdots, A_{j} \in \mathscr{A}$, and for integrable random variable $X$, let $E\left[A_{1} ; \cdots\right.$; $\left.A_{j} ; X\right]$ denote the integral $\int_{A_{1} \cap \cdots \cap A_{j}} X(\omega) P(d \omega)$. Using the Markov property at $k_{0}$, then applying (4.6), we get

$$
\frac{I_{1}\left(k_{0}\right)}{P(N(T)>n)} \leqq \frac{\kappa_{2}}{\kappa_{1}}\left(\frac{n}{n-k_{0}}\right)^{1 / 2} E\left[0<T_{k}<\mathscr{S}_{k}, 0<{ }^{\exists} k \leqq k_{0} ; T_{k_{0}}+1\right] .
$$

Since $\mathscr{S}_{k} \downarrow 0$ as $n \uparrow \infty$ for each $k$, the right-hand side tends to zero as $n \rightarrow \infty$ by the dominated convergence theorem. This proves (4.4).

Proof of (4.5) is as follows (the idea is suggested from Ritter [9]). For integers $a, b$ with $k_{0} \leqq a<b \leqq \delta n$, set

$$
A_{a+1}=\left\{T_{a+1}<\mathscr{S}_{a+1} ; N(T)>a+1\right\}
$$

and

$$
A_{k}=\left\{T_{k}<\mathscr{S}_{k} ; N(T)>k\right\} \cap\left\{\bigcap_{a<i<k}\left(A_{i}\right)^{c}\right\} \quad \text { for } \mathrm{a}+2 \leqq k \leqq b .
$$

Then, noting $T_{k}<\mathscr{S}_{k}$ on $A_{k}$ and $E\left[\mathscr{S}_{k}\right]<\infty$ by (4.3), we get

$$
I_{2}(a, b) \leqq 2 \kappa_{2} n^{-1 / 2} \sum_{a<k \leqq b} E\left[A_{k} ; \mathscr{S}_{k}+1\right] \quad \text { for } \delta<3 / 4 .
$$

Put $\kappa_{3}=\inf _{k \geqq 1}\left\{P\left(S_{k} \geqq 0\right), P\left(S_{k}<0\right)\right\}$. Then $0<\kappa_{3}(<1)$ by the central limit theorem, and we have, for $a<k \leqq b$,

$$
\begin{aligned}
& E\left[A_{k} ; \mathscr{S}_{k}\right] \leqq \kappa_{3}^{-1} E\left[A_{k} ; S_{k} \geqq 0 \quad \text { and } \quad S_{b}-S_{k} \geqq 0 \quad \text { or } \quad S_{k}<0\right. \\
& \text { and } \left.S_{b}-S_{k}<0 ; \mathscr{S}_{k}\right] \leqq \kappa_{3}^{-1} E\left[A_{k} ; \mathscr{S}_{b}\right]
\end{aligned}
$$

secause $h(x)$ is nondecreasing (resp. nonincreasing) on $(0, \infty)$ (resp. $(-\infty, 0)$ ) rom (4.3). By (4.6), (4.9), (4.10) and by $\bigcup_{a<k \leqq b} A_{k} \subset\{N(T)>a\}$, we obtain

$$
I_{2}(a, b) \leqq 2 \frac{\kappa_{2}^{2}}{\kappa_{3}}(n a)^{-1 / 2}\left\{E\left[\mathscr{S}_{b} \mid N(T)>a\right]+1\right\} \quad \text { for } \delta<3 / 4
$$

Let $c>1$ be a fixed integer. Let $r_{0}$ be the greatest of integers $r$ satisfying $\imath^{r} \leqq k_{0}$, and $r_{1}$ be the greatest of integers $r$ satisfying $c^{r} \leqq \delta n$. Then we have from (4.11) 


$$
I_{2}\left(k_{0}, \delta n\right) \leqq \sum_{r=\gamma_{0}}^{r_{1}} I_{2}\left(c^{r}, c^{r+1}\right) \leqq K^{\prime} n^{-1 / 2}\left\{J_{n, \delta}+k_{0}^{-1 / 2}\right\},
$$

where $K^{\prime}$ is a positive constant independent of $n, \delta$ and $k_{0}$,

$$
J_{n, \delta}=\sum_{r=r_{0}}^{r_{1}} \int_{-\infty}^{\infty}|x| \psi\left(n^{-1 / 2} c^{r / 2} x\right) d \theta_{c r}(x)
$$

and

$$
\theta_{n}(x)=P\left(n^{-1 / 2} S_{c n} \leqq x \mid N(T)>n\right)
$$

Finally we prove

$$
J_{n, \delta} \leqq f_{1}(\delta)
$$

for some $f_{1}(\delta)$ satisfying $f_{1}(\delta) \rightarrow 0$ as $\delta \rightarrow 0+$. To do this we need the following inequality:

For every $c \geqq 1$ there exists a constant $M=M_{c}<\infty$ such that

$$
P\left(\left|n^{-1 / 2} S_{[c n]}\right|>x \mid N(T)>n\right) \leqq M(x+1)^{-2}
$$

for every $n=1,2, \cdots$, and $x \geqq 0$, where $[a]$ is the integral part of a (see Corollary to Lemma 5.1 in Section 5). We integrate by parts and use (4.14) to get

$$
\begin{aligned}
J_{n, \tilde{o}} & \leqq M \sum_{r=r_{0}}^{r_{1}} \int_{0}^{\infty}(x+1)^{-2} d_{x}\left\{x \tilde{\psi} r\left(n^{-1 / 2} c^{f / 2} x\right)\right\} \\
& =M \int_{0}^{\infty} x(x+1)^{-3} \sum_{r=r_{0}}^{r_{1}} \tilde{\psi}\left(n^{-1 / 2} c^{r / 2} x\right) d x,
\end{aligned}
$$

where $\tilde{\psi}(x)=\psi(-x)+\psi(x)$. Set

$$
\Psi_{\delta}(x)=\frac{2}{\log c} \int_{0}^{\left(c^{\delta}\right) 1 / 2 x} y^{-1} \tilde{\psi}(y) d y \quad \text { and } \quad f_{1}(\delta)=M \int_{0}^{\infty} x(x+1)^{-3} \Psi_{\delta}(x) d x .
$$

Then it follows from (4.3) and from the convergence of (1.5) that

$$
\sum_{r=r_{0}}^{r_{1}} \tilde{\psi}\left(n^{-1 / 2} c^{r / 2} x\right) \leqq \Psi_{\delta}(x)<\infty,
$$

and hence $J_{n, \delta} \leqq f_{1}(\delta)$ and $f_{1}(\delta) \rightarrow 0$ as $\delta \rightarrow 0+$. This completes the proof.

It follows from $P\left(\sigma_{F_{h}}(Z)>0\right)=0$ that $P\left(\sigma_{F_{h}}\left(Z_{n}\right)>1\right) \rightarrow 0$ as $n \rightarrow \infty$. Moreover we can determine the rate of the convergence.

Corollary to Lemma 4.1. If $h \in \boldsymbol{C}(\boldsymbol{R} \rightarrow[0, \infty))$ satisfies the condition in Theorem, we have

$$
P\left(\sigma_{F_{h}}\left(Z_{n}\right)>1\right) \sim \pi^{-1 / 2} P\left(\sigma_{F_{h}}(\hat{Z})>1\right) e^{\mu} n^{-1 / 2} \quad \text { as } n \rightarrow \infty .
$$


Proof. Observe an obvious identity

$$
P\left(\sigma_{F_{h}}\left(Z_{n}\right)>1\right)=P\left(\sigma_{F_{n}}\left(Z_{n}\right)>1 \mid \sigma_{F_{0}}\left(Z_{n}\right)>1\right) P\left(\sigma_{F_{0}}\left(Z_{n}\right)>1\right) .
$$

Since $P\left(\sigma_{F_{0}}\left(Z_{n}\right)>1\right)=P(N(T)>n)$, we conclude (4.15) from Lemma 4.1 and (4.7).

\section{§5. Asymptotic behavior of conditional moments}

In this section we will prove the following lemma.

LEMma 5.1. Let $f$ be a real valued continuous function on $\boldsymbol{R}$ such that $f(x)=O\left(x^{2}\right)$ as $|x| \rightarrow \infty$. Then we have

$$
\lim _{n \rightarrow \infty} E\left[f\left(n^{-1 / 2} S_{[c n]}\right) \mid N(T)>n\right]=E[f(X(c))]
$$

for every $c \geqq 1$.

Here we note the following. By (1.6), we have

$$
P\left(n^{-1 / 2} S_{[c n]} \in * \mid N(T)>n\right) \stackrel{w}{\longrightarrow} P(X(c) \in *) \quad \text { in } \boldsymbol{R}
$$

for every $c \geqq 0$. Hence (5.1) is a consequence of (5.2) for bounded continuous $f$. But, if $f$ is unbounded, we cannot conclude (5.1) from (5.2) without some additional information such as the uniform integrability.

Setting $f(x)=x^{2}$ in (5.1), we get from Chebyshev's inequality the following:

Corollary to Lemma 5.1. The estimate (4.14) holds.

Before proving the lemma, we prepare several lemmas and a definition.

Lemma 5.2. If $f$ satisfies the condition in Lemma 5.1, we have

$$
\lim _{n \rightarrow \infty} E\left[f\left(n^{-1 / 2} S_{[c n]}\right)\right]=E[f(X(c))]
$$

for every $c \geqq 0$.

Proof is elementary and is omitted here.

Remark 5.3. We note that Lemma 5.1 is just reduced to Lemma 5.2, if the component random walks $\left\{S_{n}\right\}$ and $\left\{T_{n}\right\}$ are mutually independent.

Definition. Let $A(t)=\sum_{n=0}^{\infty} a_{n} t^{n}$ and $B(t)=\sum_{n=0}^{\infty} b_{n} t^{n}$ be power series with radii of convergence one, and with $|A(1-)|=|B(1-)|=\infty$. Then we write $A(t) \cong B(t)(t \rightarrow 1-)$ when $a_{n} \sim b_{n}(n \rightarrow \infty)$. 
It follows from Definition we have the following two lemmas.

Lemma 5.4. Let $A(t)$ and $C(t)=\sum_{n=0}^{\infty} c_{n} t^{n}$ be power series such that $a_{n} \sim n^{\alpha}(n \rightarrow \infty)$ for some $\alpha \geqq 0$, and such that $\sum_{n=0}^{\infty}\left|c_{n}\right|<\infty$ and $C(1) \neq 0$. Then we have $C(t) A(t) \cong C(1) A(t)(t \rightarrow 1-)$.

Lemma 5.5 (Hardy [3], 5.6, theorem 41). Suppose that $A(t) \cong(1-t)^{-\alpha}$ and $B(t) \cong(1-t)^{-\beta}(t \rightarrow 1-)$ for some positive $\alpha$ and $\beta$. Then we have $A(t) B(t) \cong(1-t)^{-\alpha-\beta}(t \rightarrow 1-)$.

Proof of Lemma 5.1. We note that the lemma follows from

$$
\lim _{n \rightarrow \infty} E\left[\left(n^{-1 / 2} S_{n}\right)^{2} \mid N(T)>n\right]=1 \quad\left(=E\left[X(1)^{2}\right]\right) .
$$

Indeed, observing the identity

$$
\left(n^{-1 / 2} S_{[c n]}\right)^{2}=\left(n^{-1 / 2} S_{n}\right)^{2}+2 n^{-1} S_{n}\left(S_{[c n]}-S_{n}\right)+\left\{n^{-1 / 2}\left(S_{[c n]}-S_{n}\right)\right\}^{2}
$$

and independence of the increments, we get (5.1) for $f(x)=x^{2}$ from (5.4) and Lemma 5.2. Then, the lemma is an easy consequence of (5.2).

To show (5.4) we introduce the following identity. Let $I(t), I_{1}(t)$ and $I_{2}(t)$ be power series given by

$$
\begin{aligned}
& I_{1}(t)=\sum_{n=1}^{\infty} t^{n} E\left[T_{n} \geqq 0 ;\left(n^{-1 / 2} S_{n}\right)^{2}\right] \\
& I_{2}(t)=\left(\sum_{n=1}^{\infty} t^{n} n^{-1 / 2} E\left[T_{n} \geqq 0 ; n^{-1 / 2} S_{n}\right]\right)^{2}
\end{aligned}
$$

and $I(t)=I_{1}(t)+I_{2}(t)$, then we have

$$
\begin{aligned}
& \sum_{n=1}^{\infty} t^{n} E\left[N(T)>n ; S_{n}^{2}\right] \\
& \quad=(1-t)^{-1 / 2} \exp \left[\sum_{n=1}^{\infty} t^{n} n^{-1}\left\{P\left(T_{n} \geqq 0\right)-1 / 2\right\}\right] I(t) \quad \text { for }|t|<1 .
\end{aligned}
$$

The proof is as follows. We recall the half-plane factorization identity: For $|t|<1$ and $-\infty<\xi, \eta<\infty$

$$
\begin{aligned}
\sum_{n=0}^{\infty} t^{n} E\left[N(T)>n ; \exp \left\{i\left(\xi S_{n}+\eta T_{n}\right)\right\}\right] \\
\quad=\exp \left[\sum_{n=1}^{\infty} t^{n} n^{-1} E\left(T_{n} \geqq 0 ; \exp \left\{i\left(\xi S_{n}+\eta T_{n}\right)\right\}\right)\right] .
\end{aligned}
$$

The formula (5.6) is a special case of Mogul'skii and Pecherskii [8], Theorem 1 in which we take the dimension $m=2$ and the semigroup $H=$ $\{(x, y) ; y \geqq 0\}$. See also Corollary 3 and remark in [8], §2, from which we have $E_{0, n}=\left\{T_{n} \geqq 0\right\}$ in our case. Differentiating (5.6) with respect to $\xi$ twice, and then letting $\xi=\eta=0$, we get (5.5).

Let us prove

$$
I(t) \cong 2^{-1} \sum_{n=0}^{\infty} t^{n}=2^{-1}(1-t)^{-1} \quad(t \rightarrow 1-) .
$$


By a two-dimensional modification of Lemma 5.2, we have

$$
E\left[T_{n} \geqq 0 ; n^{-1 / 2} S_{n}\right] \longrightarrow E[Y(1) \geqq 0 ; X(1)]=0
$$

and

$$
E\left[T_{n} \geqq 0 ;\left(n^{-1 / 2} S_{n}\right)^{2}\right] \longrightarrow E\left[Y(1) \geqq 0 ; X(1)^{2}\right]=1 / 2
$$

as $n \rightarrow \infty$. Then

$$
I_{1}(t) \cong 2^{-1} \sum_{n=0}^{\infty} t^{n}=2^{-1}(1-t)^{-1} \quad(t \rightarrow 1-)
$$

by (5.9), and the coefficients of the power series $I_{2}(t)$ converge to zero by (5.8), which proves (5.7).

Finally we note that

$$
\sum_{n-1}^{\infty} n^{-1}\left|P\left(T_{n} \geqq 0\right)-1 / 2\right|<\infty
$$

(Rosén [10]). Then we have from Lemmas 5.4, 5.5 and from (5.7)

$$
\text { the right-hand side of }(5.5) \cong 2^{-1} e^{\mu}(1-t)^{-3 / 2} \quad(t \rightarrow 1-),
$$

where $\mu$ is given by (4.8). Hence we have

$$
E\left[N(T)>n ; S_{n}^{2}\right] \sim 2^{-1} e^{\mu}(-1)^{n}\left(\begin{array}{c}
-3 / 2 \\
n
\end{array}\right) \sim \pi^{-1 / 2} e^{\mu} n^{1 / 2} \quad(n \rightarrow \infty) .
$$

Combining this formula with (4.7), we conclude (5.4). This completes the proof.

\section{REFERENCES}

[1] P. Billingsley, Convergence of probability measures, Wiley, New York, 1968.

[2] E. Bolthausen, On a functional central limit theorem for random walks conditioned to stay positive, Ann. Probab., 4 (1976), 480-485.

[ 3 ] G. H. Hardy, Divergent series, Clarendon Press, Oxford, England, 1949.

[4] D. L. Iglehart, Functional central limit theorems for random walks conditioned to stay positive, Ann. Probab., 2 (1974), 608-619.

[ 5 ] K. Ito and H. P. McKean, Jr., Diffusion processes and their sample paths, second printing, Springer, Berlin, 1974.

[6] M. V. Kozlov, On the asymptotic behavior of the probability of non-extinction for critical branching processes in a random environment, Theory Probab. Appl., 21 (1976), 791-804.

[ 7 ] T. Lindvall, Weak convergence of probability measures and random functions in the function space $D[0, \infty)$, J. Appl. Probab., 10 (1973), 109-121.

[ 8 ] A. A. Mogul'skii and E. A. Pecherskii, On the first exit time from a semigroup in $\boldsymbol{R}^{m}$ for a random walk, Theory Probab. Appl., 22 (1977), 818-825.

[9] G. A. Ritter, Growth of random walks conditioned to stay positive, Ann. Probab., 9 (1981), 699-704. 
[10] B. Rosén, On the asymptotic distribution of sums of independent identically distributed random variables, Ark. Mat., 4 (1962), 323-332.

[11] M. Shimura, A class of conditional limit theorems related to ruin problem, Ann. Probab., 11 (1983), 40-45.

[12] F. Spitzer, A Tauberian theorem and its probability interpretation, Trans. Amer. Math. Soc., 94 (1960), 150-169.

[13] D. Williams, Decomposing the Brownian path, Bull. Amer. Math. Soc., 76 (1970), 871-873.

Institute of Mathematics

University of Tsukuba

Sakura-mura Niihari-gun

Ibaraki, 305 Japan 